\title{
Facial Nerve Schwannoma Arising From the Cerebellopontine Angle -Case Report-
}

\author{
Toshiyuki Amano, ${ }^{1}$ Yuhei SAngatsudA, ${ }^{1}$ Nobuhiro HAtA, ${ }^{1}$ Daisuke InOUe, ${ }^{1}$ \\ Megumu MORI, ${ }^{1}$ Akira NAKAMIZO, ${ }^{1}$ Koji YOSHIMOTO, ${ }^{1}$ \\ Masahiro MIZOGUCHI, ${ }^{1}$ and Tomio SASAKI ${ }^{1}$
}

${ }^{1}$ Department of Neurosurgery, Graduate School of Medical Sciences, Kyushu University, Fukuoka, Fukuoka

\begin{abstract}
A 74-year-old female suffered hearing disturbance in the right ear persisting for several years, followed by sudden onset of right facial nerve palsy. Her symptoms gradually worsened and neuroradiological imaging revealed a 4-cm cerebellopontine angle (CPA) tumor. Intraoperatively, the tumor was found to originate from the facial nerve. Total removal of the tumor was achieved, followed by a split hypoglossal-facial nerve anastomosis. Facial nerve schwannomas are rare and extremely difficult to preoperatively distinguish from vestibular schwannomas, especially if arising from the CPA and the internal auditory canal. However, preoperative diagnosis of facial nerve schwannomas is important because functional preservation of the facial nerve is more challenging than for vestibular schwannomas. Facial nerve palsy is one of the most unique symptoms in patients with facial nerve schwannomas, but is rare with vestibular schwannomas. Facial nerve schwannomas should be included in the differential diagnosis of CPA tumors with atypical clinical manifestations, and patients should be informed before surgery of the possibility of facial nerve dysfunction and the consequent need for facial nerve graft or reconstruction.
\end{abstract}

Key words: facial nerve schwannoma, cerebellopontine angle, facial palsy

\section{Introduction}

Intracranial schwannomas are benign, slowly growing tumors accounting for $6 \%$ to $8 \%$ of all primary intracranial tumors. Almost all intracranial schwannomas arise from the vestibular nerve, followed by the trigeminal nerve. The prevalence of facial nerve schwannomas has been estimated to be less than $2 \%$ of intracranial schwannomas. ${ }^{8)}$ Facial nerve schwannomas might arise from any segment of the facial nerve, but commonly involve the geniculate ganglion, and can be visualized as an enhanced lesion in the middle cranial fossa. However, if the tumor is limited to the cerebellopontine angle (CPA) or the internal auditory canal (IAC), facial nerve schwannomas are often impossible to distinguish from vestibular schwannomas by neuroradiological imaging. Facial weakness and hearing disturbance have been reported as initial symptoms in more than half of patients with facial nerve schwannomas. Other symptoms include tinnitus and ear canal pain., ${ }^{3,7,9}$ Hearing disturbance is a common symptom with tumors such as vestibular schwannomas or CPA meningiomas. On the other hand, facial weakness is a rare symptom for

Received April 10, 2012;

Accepted May 6, 2012 such tumors, even if relatively large, and facial weakness is more commonly attributable to facial nerve schwannoma. We report here a rare case of facial nerve schwannoma limited to the CPA.

\section{Case Report}

A 74-year-old female suffered hearing difficulty in the right ear persisting for several years. Two months before admission, sudden onset of facial weakness began and gradually worsened to House-Brackmann grade $\mathrm{V}$ on admission. Neuro-otological examination revealed deafness in the right ear without any reproducible waves in the auditory brainstem response (ABR). Computed tomography (CT) showed a 4-cm multicystic tumor with intratumoral hemorrhage and heterogeneous enhancement with contrast medium in the right CPA (Fig. 1A, B). Bone window CT detected no enlargement of either the IAC or the facial nerve canal (Fig. 1C). Magnetic resonance (MR) imaging showed the lesion as hypointense on $\mathrm{T}_{1}$-weighted images with heterogeneous enhancement after intravenous administration of gadolinium, without any enhanced lesions in the facial nerve canal or the middle cranial fossa (Fig. $2 \mathrm{~A}, \mathrm{~B}) . \mathrm{T}_{2}$-weighted MR imaging revealed the lesion as an 

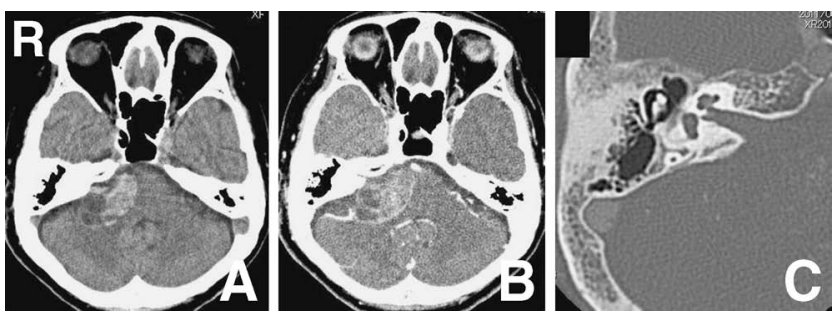

Fig. 1 A, B: Computed tomography (CT) scans without (A) and with (B) contrast medium demonstrating a multicystic tumor with intratumoral hemorrhage at the cerebellopontine angle. C: Bone window CT scan showing no enlargement of the internal auditory canal.
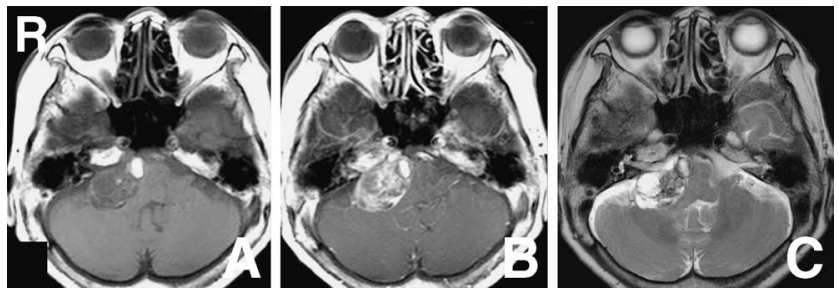

Fig. 2 A, B: $T_{1}$-weighted magnetic resonance (MR) images showing an isointense mass $(A)$ and irregular enhancement by gadolinium (B) in the right cerebellopontine angle without enhancement of the geniculate ganglion or middle cranial fossa. C: $T_{2}$-weighted MR image showing an intratumoral hypointense lesion associated with hemorrhage.
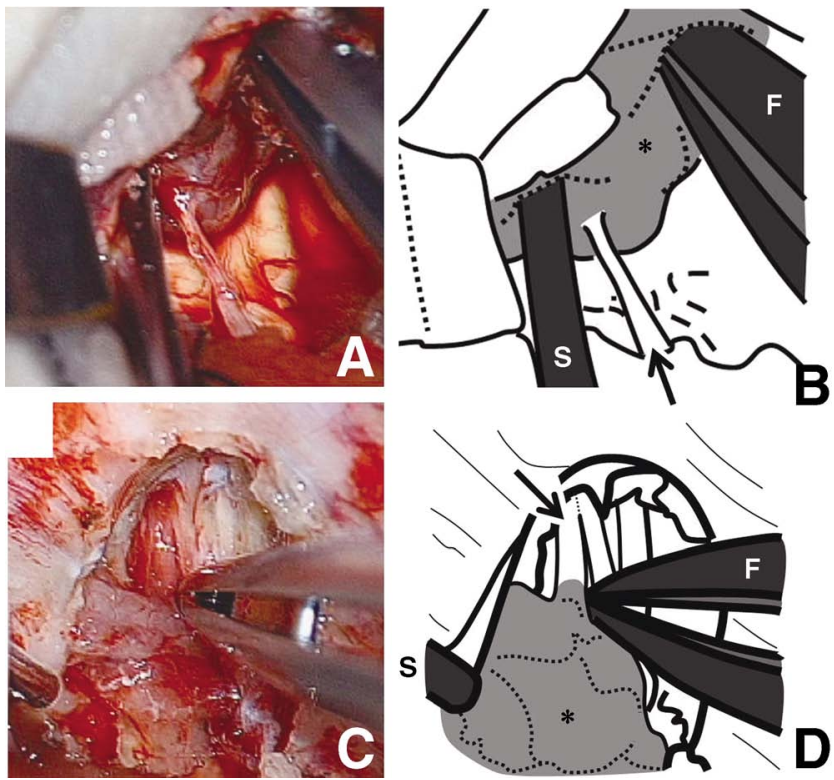

Fig. 3 A, B: Intraoperative photograph (A) and schema (B) showing the proximal facial nerve (arrow) suddenly disappearing on the surface of the tumor (asterisk). C, D: Intraoperative photograph (C) and schema (D) after opening the internal auditory meatus showing the tumor (asterisk) originating from the distal facial nerve (arrow) and the intact superior vestibular nerve along the facial nerve. F: forceps, S: suction probe. almost hyperintense mass with a hypointense center, which may suggest intratumoral hemorrhage (Fig. 2C).

Surgical resection of the tumor was performed through a right suboccipital retrosigmoid approach. During the operation, we observed that cranial nerve VIII was fanned out on the surface of the tumor, whereas cranial nerve VII suddenly disappeared on the surface of the tumor, leading to the identification of schwannoma originating from the facial nerve (Fig. 3A, B). After opening the internal auditory meatus, the facial nerve was identified by facial motor evoked potential and the schwannoma identified as originating from the facial nerve, whereas the superior vestibular nerve remained intact (Fig. 3C, D). During the operation, we could not detect any responses during facial nerve stimulation at the root exit zone of the facial nerve, so total resection of the tumor was performed. Three months later, she received a split hypoglossal-facial nerve anastomosis and her facial nerve function gradually recovered. Seven months later, her facial nerve function was evaluated as House-Brackmann grade V, and further functional recovery might be expected. Histological examination demonstrated both fascicular proliferation of spindle-shaped tumor cells and loose areas with many foamy macrophages and hemosiderin deposition, which are indicative of schwannoma.

\section{Discussion}

Facial nerve schwannoma is a relatively rare tumor arising from the labyrinthine, geniculate, tympanic, or vertical segments of the facial nerve. Involvement of the geniculate ganglion is the most common, accounting for $44 \%$ to $97 \%$ of cases, and CPA involvement ranges from $24 \%$ to $53 \% .^{3,7,9,10)}$ More than $93 \%$ of facial nerve schwannomas involve three or more segments of the facial nerve, and cases limited to the CPA are extremely rare, accounting for less than $5 \%$ of facial nerve schwannomas. ${ }^{1)}$ In addition to their rarity, CPA facial nerve schwannomas are often indistinguishable from vestibular schwannomas until operative intervention. ${ }^{9)}$

CPA tumors may manifest as various clinical symptoms. Hearing disturbance is the most common symptom in patients with CPA tumors, including facial nerve schwannomas. More than half of patients with facial nerve schwannomas presented with hearing loss. ${ }^{5,7,10)}$ On the other hand, facial weakness is the most important and unique clinical symptom, found in $57 \%$ to $63 \%$ of patients with facial nerve schwannomas, but only $5 \%$ to $7 \%$ of patients with vestibular schwannomas, even large ones. ${ }^{5,7,9)}$ These findings strongly suggest that facial nerve palsy associated with CPA tumors might be indicative of facial nerve schwannomas. Another unique clinical symptom associated with facial nerve schwannomas is facial spasm, reported in $2 \%$ to $27 \%$ of patients, as compared with $0.6 \%$ of patients with vestibular schwannomas. ${ }^{4,7,9)}$ We should carefully consider facial nerve schwannomas in the differential diagnosis of CPA tumors, especially in patients with atypical clinical manifestations.

MR imaging might be the most important neurological examination for the diagnosis of facial nerve schwanno- 
mas. MR imaging with gadolinium can identify enhancement of the facial canal or the geniculate ganglion in the middle cranial fossa in cases of facial nerve schwannomas involving the geniculate ganglion. ${ }^{2,8-10)}$ Without this distinction, this rare tumor is very difficult to preoperatively diagnose as facial nerve schwannoma, even with small tumors. We should carefully consider enhanced lesions in the middle cranial fossa when assessing CPA tumors with MR imaging, especially in cases with atypical clinical presentation.

As with vestibular schwannomas, facial nerve schwannomas can be cured by radial resection, but surgical outcomes seem to be quite different. Vestibular schwannomas compress the facial nerve to varying degrees at all times, but functional preservation of the facial nerve can be achieved in almost all cases by subcapsular or subperineurial dissection, even with remarkable fanning and large tumors. ${ }^{6)}$ In contrast, functional preservation of the facial nerve is relatively challenging even with very small facial nerve schwannomas. In our case, the facial nerve was not fanning at all but suddenly disappeared on the surface of the tumor, with barely visible cochlear and vestibular nerves. Intraoperative microphotographs definitively showed the tumor as originating from the facial nerve. Since the patient's facial nerve function was already lost, there was no opportunity for preservation, and she subsequently received a split hypoglossal-facial nerve anastomosis.

Direct facial nerve anastomosis, whether using interpositional graft or not, require an adequate length of a bundle of facial nerves. In our case, facial nerve schwannoma extended to the fundus in the IAC, so a sufficient length of a bundle of facial nerves could not be preserved. On the other hand, split hypoglossal-facial nerve anastomosis is occasionally performed for patients suffering severe facial palsy, expecting satisfying recovery of the facial nerve function. ${ }^{11)}$ Although our patient finally agreed to receive another operation 3 months after facial nerve schwannoma surgery, earlier facial nerve anastomosis might have provided a better functional outcome for the facial nerve. As with our patient, patients with facial nerve schwannomas often require facial nerve graft or reconstruction. The optimal timing of surgical treatment for facial nerve schwannomas is still controversial and gamma knife surgery might be required in some cases.

Functional preservation of the facial nerve is relatively difficult after radical resection of facial nerve schwannoma. Patients with facial nerve schwannomas should be informed of the relative risk of facial nerve dysfunction and the possible need for facial nerve graft or reconstruction before surgery. Therefore, we have to establish the preoperative diagnosis of this rare tumor as facial nerve schwannoma. Although facial nerve schwannoma limited to the CPA is extremely rare, the clinical manifestations and neuroradiological examinations can assist in the diagnosis of tumors suspected to be facial nerve schwannoma. We should pay more attention to the possibility of facial nerve schwannoma with CPA tumors, especially those with manifestations that are atypical for vestibular schwannomas.

\section{Conflicts of Interest Disclosure}

The authors have no personal financial or institutional interest in any of the drugs, materials, or devices in the article. All authors who are members of The Japan Neurosurgical Society (JNS) have registered online Self-reported COI Disclosure Statement Forms through the website for JNS members.

\section{References}

1) Brackmann DE, Bartels LJ: Rare tumors of the cerebellopontine angle. Otolaryngol Head Neck Surg 88: 555-559, 1980

2) Fabiano AJ, Plunkett RJ, Gibbons KJ: Diagnosis of facial nerve schwannoma by magnetic resonance imaging enhancement of the geniculate ganglion. Arch Neurol 67: 112-113, 2010

3) Günther M, Danckwardt-Lillieström N, Gudjonsson O, Nyberg G, Kinnefors A, Rask-Andersen H, Ekvall L: Surgical treatment of patients with facial neuromas-a report of 26 consecutive operations. Otol Neurotol 31: 1493-1497, 2010

4) Matthies C, Samii M: Management of vestibular schwannomas (acoustic neuromas): the value of neurophysiology for evaluation and prediction of auditory function in 420 cases. Neurosurgery 40: 919-930, 1997

5) Samii M, Gerganov V, Samii A: Improved preservation of hearing and facial nerve function in vestibular schwannoma surgery via the retrosigmoid approach in a series of 200 patients. J Neurosurg 105: 527-535, 2006

6) Sasaki T, Shono T, Hashiguchi K, Yoshida F, Suzuki SO: Histological considerations of the cleavage plane for preservation of facial and cochlear nerve functions in vestibular schwannoma surgery. J Neurosurg 110: 648-655, 2009

7) Sherman JD, Dagnew E, Pensak ML, van Loveren HR, Tew JM Jr: Facial nerve neuromas: report of 10 cases and review of the literature. Neurosurgery 50: 450-456, 2002

8) Symon L, Cheesman AD, Kawauchi M, Bordi L: Neuromas of the facial nerve: a report of 12 cases. Br J Neurosurg 7: 13-22, 1993

9) Thompson AL, Aviv RI, Chen JM, Nedzelski JM, Yuen HW, Fox AJ, Bharatha A, Bartlett ES, Symons SP: Magnetic resonance imaging of facial nerve schwannoma. Laryngoscope 119: 2428-2436, 2009

10) Wiggins RH 3rd, Harnsberger HR, Salzman KL, Shelton C, Kertesz TR, Glastonbury CM: The many faces of facial nerve schwannoma. AJNR Am J Neuroradiol 27: 694-699, 2006

11) Yetiser S, Karapinar U: Hypoglossal-facial nerve anastomosis: a meta-analytic study. Ann Otol Rhinol Laryngol 116: 542-549, 2007

Address reprint requests to: Toshiyuki Amano, MD, Department of Neurosurgery, Graduate School of Medical Sciences, Kyushu University, 3-1-1 Maidashi, Higashi-ku, Fukuoka 812-8582, Japan.

e-mail: amano@ns.med.kyushu-u.ac.jp 